\title{
Evaluation of Increased Sensitivity of Morning Bleach Sample for Detection of Acid Fast Bacilli in Pulmonary Samples
}

\author{
Pranali Pingle $^{1 *}$, Pradeep Apte1, Rakesh Trivedi² \\ ${ }^{1}$ Medicare Hospital and Research Centre, Indore, India \\ ${ }^{2}$ P.M.B. Gujarati Science College, Indore, India \\ Email: pranalispingle@yahoo.com, aptep@yahoo.com
}

Received 6 June 2014; revised 7 July 2014; accepted 21 July 2014

Copyright (C) 2014 by authors and Scientific Research Publishing Inc.

This work is licensed under the Creative Commons Attribution International License (CC BY).

http://creativecommons.org/licenses/by/4.0/

(c) (i) Open Access

\section{Abstract}

Around 85\% of the cases of Tuberculosis (TB) are pulmonary in origin and the routine diagnosis usually depends on sputum microscopy. The conventional direct Ziehl-Nelson (ZN) staining technique has been found to have a low sensitivity. The main objective of the study was to verify whether the bleach concentration method increases the sensitivity of sputum smear microscopy for AFB or not and also to see whether the first single morning sample alone is sufficient and better than the three pooled samples after bleach concentration followed by $\mathrm{ZN}$ staining. A total of 365 samples were studied from 131 clinically suspected cases of pulmonary TB which included sputum (112), gastric aspirate (5), endotracheal tube washing (2), and bronchial lavage (12). All these samples were processed for conventional ZN staining and Bleach concentration method followed by ZN staining. An increase in positivity was observed in all the cases after using the bleach concentration method and the most significantly useful was that in the case of first morning sputum samples where it increased from $11.6 \%$ to $41.96 \%$. Bleach concentration is a simple, cheap and easily available method and also very safe because it kills the Mycobacteria in the process. Its positivity rate is better as compared to direct conventional ZN staining.

\section{Keywords}

Pulmonary Tuberculosis, M. tuberculosis, Bleach Method

\footnotetext{
${ }^{*}$ Corresponding author.
} 


\section{Introduction}

Tuberculosis (TB) is one of the leading chronic infectious diseases throughout the world accounting for about 8.8 million incident cases in 2010 [1]. The latest estimates included in this report are that there were almost 9 million new cases in 2011 and 1.4 million TB deaths (990,000 among HIV negative people and 430,000 HIVassociated TB deaths). This is despite the availability of treatment that will cure most cases of TB [2]. Its etiological agent Mycobacterium tuberculosis has accounted for more human deaths than any other pathogen [3]. Tuberculosis usually attacks the lungs but can also affect other parts of the body. It is spread through the air when people who have an active MTB infection cough, sneeze, or otherwise transmit their saliva through the air [4]. Though India is the second-most populous country in the world, India has more new TB cases annually than any other country. In 2009, out of the estimated global annual incidence of 9.4 million TB cases, 2 million were estimated to have occurred in India, thus contributing to a fifth of the global burden of TB. It is estimated that about $40 \%$ of Indian population is infected with TB bacillus. The incidence of TB in India is estimated based on findings of the nationwide [5].

Tuberculosis usually attacks the lungs but can also affect other parts of the body. It is spread through the air when people who have an active MTB infection cough, sneeze, or otherwise transmit their saliva through the air.

Pulmonary TB is the most frequent cause of death by an infectious agent worldwide. The unchecked epidemics of Tuberculosis (TB) and HIV remain two of the greatest global challenges to medicine and public health. The diagnosis of TB remains clinically challenging and logistically difficult in resource-poor settings. Microscopy and culture methods are the main TB diagnostics used in resource-poor settings. Both require good sputum samples which are not always easy to obtain. Sputum is a heterogeneous sample and can be difficult to manipulate and the excretion of TB bacilli varies by individual.

Despite recent advances in Mycobacteriology, early laboratory diagnosis of TB still relies on examination of stained smears. But the technique's sensitivity is not optimal, ranging from $9 \%-46 \%$ when used in control programs. In the last decade, many researchers have suggested that the performance of smear microscopy can be significantly improved if sputum is liquefied with one or another chemical reagent and there after concentrated with centrifugation prior to acid fast staining [6] [7]. There are various concentration methods for improving sensitivity of direct microscopy for detection of AFB of which the bleach (Sodium hypochlorite, $\mathrm{NaOCl}$ ) concentration method is the safest, inexpensive and easy to perform [8]. The bleach method can improve case detection of Pulmonary TB with a maintained specificity [9]-[11]. The bleach method improves detection of AFB in sputum specimen, thereby improving case finding of Pulmonary TB [2] [8] [12]-[16]. As potent disinfectant bleach, unlike other concentration methods, also kills mycobacteria and thus eliminates the risk of laboratory infection. The technique is safe, inexpensive and easy to perform [8]. Main disadvantage is bleach treated specimens cannot be used for mycobacterial cultures since it kills M. Tuberculosis [17] [18]. An increase in positivity using the bleach concentration has been observed by many workers [15] [19] [20]. Increased positivity by bleach concentration have also suggested that it could be possible to do a diagnosis of TB using a single sample instead of three day successive samples as has been conventionally done [14] [17] [19]. It is already known that the increase in detection with the third sputum sample is only $2 \%-5 \%$, meaning that the collection of three samples is unnecessary. This would mean that if only a single sample is used it would considerably reduce the workload in busy laboratories. Collection of a single sample may improve patient compliance and reduce cost. None of these studies have compared the increased positivity after bleach concentration using 3-day pooled samples and a single first morning sample. Based on the above literature we have used the bleach concentration method in pulmonary specimens in the present study.

The main objectives of this study are as follows:

1) To evaluate and compare the possibility of using a single first morning sputum sample instead of three samples in light of the bleach concentration method;

2) Application of the bleach method to improve smear microscopy for the diagnosis of pulmonary TB using different sample sources;

3) To re-evaluate the sensitivity of the bleach method followed by ZN staining by comparing it with the conventional direct ZN staining.

\section{Materials and Methods}

A study of 131 cases of suspected tuberculosis was carried on samples that consisted of sputum (112), gastric 
aspirates (5), endotracheal washing (2) and bronchial lavage (12). All samples were collected from the patients coming to Medicare Hospital as out or in patients. The sputum samples were grouped into two as pooled 3 day and a single first morning sample. Smears from both the groups were first directly stained by the conventional direct ZN staining method. Then samples from both groups were subjected to the bleach concentration method which was followed by ZN staining. To compare the increased positivity between smears a separate lot of 11 sputum samples which were positive by direct staining were subjected to bleach concentration followed by staining.

The bleach concentration method was performed using a slight modification of the original method [21]. Sterile, disposable test tubes were used throughout the procedure. Approximately $1-2 \mathrm{ml}$ of specimen was mixed with equal amount of commercially available $4 \%$ sodium hypochlorite (Merck). After thorough mixing, the mixture was incubated for 15 minutes at room temperature with frequent mixing at intervals (A prolonged exposure and a higher concentration of bleach solution reduces the possibility of detecting AFB [22]). An equal volume of commercially available distilled water was added and mixed thoroughly using a vortex mixer followed by centrifugation at $3000 \mathrm{rpm}$ for 15 minutes. The supernatant was discarded and smears were prepared using one drop of the sediment, air dried, heat fixed and stained by ZN staining. The direct ZN stained smear and bleach processed ZN smears were then examined microscopically under $100 \times$ oil fields for the presence of AFB which is the standard procedure. As a control $2 \mathrm{ml}$ of distilled water was centrifuged and the sediment was stained by ZN staining to rule out any error due to contamination while testing each specimen. The findings of all the above studies were then compared and correlated.

\section{Observations}

The cases used in this study covered a wide age group ranging from infants to adults and included both males and females. The total 365 samples studied consisted of sputum $(n=336(112 \times 3$ samples each $))$, gastric aspirate $(n=15(5 \times 3$ samples each)), endotracheal tube washing $(n=2)$ and bronchial lavage $(n=12)$. The details of different types of specimens that were collected and their findings are shown in Table 1.

In the group of 112 three day pooled sputum samples 16 were positive by direct ZN staining and the positivity increased to 32 after bleach concentration. In the second group of single first morning samples the positivity increased from 13 to 47 when the direct and bleach processed morning samples were compared.

All the 5 gastric aspirate samples were negative by direct ZN staining, but after bleach concentration 2 were positive for AFB. The 2 endotracheal tube washing samples were negative by direct staining, but after bleach concentration both were positive for AFB. Of the 12 bronchial lavage samples only one was positive by direct staining, but after bleach concentration 5 were positive for AFB.

The positivity of AFB was also compared between the smears for direct staining and those processed by bleach concentration. All the 11 direct smear positive sputum samples which were in this study showed increased positivity with varying degrees depending on their initial positivity. Four samples showed scanty bacilli whose positivity rose to $1+$ to $2+$ after bleach concentration. Four were $1+$ positive which became $2+$ to $3+$ after bleach concentration and three samples which were $2+$ positive became $3+$ after bleach concentration. Fourteen pooled sample smears which were negative by direct ZN staining gave a positivity ranging from scanty $(n=7)$,

Table 1. Comparison of pulmonary samples treated by direct ZN staining and bleach concentration followed by ZN staining.

\begin{tabular}{|c|c|c|c|}
\hline Specimen & Number & $\begin{array}{c}\text { Positive by conventional } \\
\text { ZN staining }\end{array}$ & $\begin{array}{c}\text { Positive on bleach concentration } \\
\text { method }\end{array}$ \\
\hline Pooled samples & & $16(14.28 \%)$ & $32(28.57 \%)$ \\
\hline sputum & 112 & & \\
\hline Morning samples & & $13(11.60 \%)$ & 47 (41.96\%) \\
\hline Gastric aspirate & 5 & 0 & $2(40 \%)$ \\
\hline E T Tube & 2 & 0 & $2(100 \%)$ \\
\hline Bronchial lavage (BAL) & 12 & $1(8.33 \%)$ & $5(41.66 \%)$ \\
\hline Total & 131 patients & $17(16+1)$ & $56(47+2+2+5)$ \\
\hline
\end{tabular}

Pooled and morning samples were treated by ZN staining and bleach concentration followed by ZN staining method. Out of total sample of 112 , the number of positive samples by both methods has been shown, other samples were reported negative. 
$1+(n=4)$ and $2+(n=3)$ after bleach concentration. Twenty-seven first morning sample smears which were negative by direct ZN staining gave a positivity ranging from scanty $(n=18), 1+(n=5), 2+(n=4)$ after bleach concentration.

\section{Discussion}

The main aim of this study was to compare the improvement in positivity after bleach concentration on 3-day pooled and a single first morning sputum sample. On searching the literature till date it was observed that though the bleach concentration method for increased positivity has been used on pulmonary samples in the past but this is the first study where two groups of sputum samples (three day pooled and first morning) were compared respectively and with the direct ZN staining.

In the present study in Group I $14.28 \%(\mathrm{n}=16)$ were positive by direct $\mathrm{ZN}$ staining which is more as compare to study by Iqbbal S. et al. [23], 2011 (12.20\%) where the samples were tested and compared by ZN staining, LJ culture and PCR method Mutha A. et al. [9], 2005 (5.38\%) and Angaw B. et al. [11], 2012 (12.5\%) of AFB smears positive for Tuberculosis. However, the results of our study were lower than what reported by Negi S. S. et al. [24], 2005 where they had 33.79\% positivity by ZN staining obtained from 156 patients for the evaluation of pulmonary and extrapulmonary TB and compared ZN staining, BACTEC culture and PCR. While in Group II $11.60 \%(n=13)$ were positive by direct $\mathrm{ZN}$ staining in only morning samples which can be only compared by the study done by Angaw B. et al. [11], 2012. In this 264 samples were collected and processed by ZN staining and bleach concentration method, Out of the three consecutive sputum specimens collected from the same patient, the early morning sputum specimen showed a higher rate of positive smear results as compared to the two spot specimens (12.5\%) by direct ZN staining which is more as compared to our study.

With the use of Bleach concentration method in Group I, we were able to detect M. tuberculosis in $28.57 \%$ (n = 32) which is more as compared to the study by Mutha A. et al. [9], 2005 (9.09\%), Mindloi P. B. et al. [25], 2013 (23.14\%) and Angaw B. et al. [11], 2012 (23.2\%). However, the study by Makunde M. H. et al. [12], 2007 and James A. et al. [26], 2013 reported (28.3\% and 59.7\%), positive by Bleach concentration method. While in Group II the positivity increased to $41.96 \%(\mathrm{n}=47)$ which is more as compared to the study Angaw $\mathrm{B}$. et al. [11], 2012 in which the bleach concentration performed on morning sample showed (23.2\%) positivity.

In many pediatric and geriatric cases or whenever no expectoration was possible gastric aspirate, endotracheal tube washings or a bronchial lavage was used for analysis [5] [27]. An increase in positivity in all the three sample types was observed by bleach concentration method. The increase being $40 \%$ in gastric aspirates, $100 \%$ in endotracheal tube washings and from $8.33 \%$ to $41.66 \%$ in bronchial lavages (Table 1). However, to best of my knowledge, this is the first study in which bleach concentration method is tried in gastric aspirates, endotracheal tube washings and bronchial lavage and the results shows improved detection of AFB. Although the more research work should be done in this field.

The major advantage of the bleach-concentration method is the higher density of bacilli per microscope filed obtained and the reduction of debris present in the sputum with a potential to increase sensitivity. The limitation of this study was that the samples were not processed for culture. Even though the concentration technique is slightly more labor intensive compared to the direct method as it needs a centrifuge; it has a higher detection rate and a potentially lower risk of laboratory associated transmission.

Statistical evaluation of the significant differences in the two types of sample groups used in sputum and in the overall study was done using the Z-test (Table 2). It can be clearly from this table that:

1) There is no significant difference on direct $Z N$ staining in the 3-day pooled and first morning sputum groups;

2) On bleach concentration there is a significant difference between the 3-day pooled and first morning groups;

3) The increase in positivity in the 3 day pooled samples group after bleach concentration highly significant;

4) The increase in positivity in the first morning sample group after bleach concentration is highly significant;

5) The increase in positivity in the overall study is highly significant after bleach concentration when compared with direct ZN staining.

\section{Conclusion}

By this study, it can be concluded that compared to pooled bleach sputum sample a single first morning speci- 
Table 2. Statistical data analysis.

\begin{tabular}{cccccccc}
\hline S.N & Group 1 & N1 & Group 2 & N2 & Z Test Value & p Value & Result \\
\hline 1 & POOLED ZN & 16 & MORNING ZN & 13 & 0.59 & $\mathrm{p}>0.05$ & NOT SIG \\
2 & POOLED BLEACH & 32 & MORNING BLEACH & 47 & 2.06 & $\mathrm{p}<0.05$ & SIG \\
3 & POOLED ZN & 16 & POOLED BLEACH & 32 & 2.6 & $\mathrm{p}<0.01$ & H.SIG \\
4 & MORNING ZN & 13 & MORNING BLEACH & 47 & 5.12 & $\mathrm{p}<0.01$ & H.SIG \\
$\mathbf{5}$ & Total ZN & $\mathbf{1 7}$ & Total Bleach & $\mathbf{5 6}$ & $\mathbf{5 . 3 7}$ & $\mathbf{p}<\mathbf{0 . 0 1}$ & H.SIG
\end{tabular}

The significant difference of two sample populations has been calculated by $\mathrm{Z}$ test.

men is enough to give a conclusive diagnosis of pulmonary tuberculosis which would save a lot of time and early treatment could be initiated. And it can be said that the bleach concentration method, as is re-evident from this study, is a cost effective, sensitive versatile and safe procedure for the demonstration of AFB. It can be a valuable in using it as diagnostic tool regardless of the specimen type or source used.

\section{References}

[1] WHO (2011) The Sixteenth Global Report on Tuberculosis. WHO, Geneva.

[2] Githui, W.A., Matu, S.W., Muthmi, L.N. and Juma, E. (2007) Improved Diagnosis of Ziehl Neelsen Smear Negative Tuberculosis Using Sodium Hypochlorite Sedimentation Method. East African Medical Journal, 84, 455-459.

[3] WHO (2012) Global Tuberculosis Report. WHO, Geneva.

[4] TB India (2012) Revised National TB Control Programme, Annual Status Report. Central TB Division, Directorate General of Health Services, Ministry of Health and Family Welfare, NirmanBhawan, New Delhi.

[5] Rahbar, M. and Hajia, M. (2007) Value of Gastric Lavage for Diagnosis of Pulmonary Tuberculosis. Pakistan Journal of Medical Sciences, 23, 51-53.

[6] Angeby, K. (2004) Tuberculosis. Diagnosis and Drug Susceptibility Testing Where Resources Are Scarce. Karolinska University Press, Stockholm.

[7] Cattamanchi, A., Davis, J.L., Pai, M., Huang, L., Hopewell, P.C. and Steingert, K.R. (2010) Does Bleach Processing Increase the Accuracy of Sputum Smear Microscopy for Diagnosing Pulmonary Tuberculosis? Journal of Clinical Microbiology, 48, 2433-2439. http://dx.doi.org/10.1128/JCM.00208-10

[8] Gebre, N.U., Karlsson, G., Jonsson, R., et al. (1995) Improved Microscopical Diagnosis of Pulmonary Tuberculosis in Developing Countries. Transactions of the Royal Society of Tropical Medicine and Hygiene, 89, 191-193. http://dx.doi.org/10.1016/0035-9203(95)90491-3

[9] Mutha, A., Tiwari, S., Khubnanai, H. and Mall, S. (2005) Application of Bleach Method to Improve Sputum Smear Microscopy for the Diagnosis of Pulmonary Tuberculosis. Indian Journal of Pathology and Microbiology, 48, 513517.

[10] Steingert, K.R., Ng, V., Henry, M., et al. (2006) Sputum Processing Methods to Improve the Sensitivity of Smear Microscopy for Tuberculosis: A Systemic Review. The Lancet Infectious Diseases, 6, 664-674. http://dx.doi.org/10.1016/S1473-3099(06)70602-8

[11] Angaw, B., Mulu, A., Abate, E., Belay, T., et al. (2012) Improved Detection of Acid Fast Bacilli in Sputum by BleachConcentration Technique at Gondar University Teaching Hospital, Northwest Ethiopia. Ethiopian Medical Journal, 50, 349-354.

[12] Makunde, W.H., Makunde, R.A., Kamugisha, L.M., Mgema, S.G. and Liwa, A. (2007) Improved Microscopy Diagnosis of Pulmonary Tuberculosis Using Sodium Hypochlorite Concentration Technique in Tanga, Tanzania. Tanzania Health Research Bulletin, 9, 87-93. http://dx.doi.org/10.4314/thrb.v9i2.14309

[13] Merid, Y., Yassin, M.A., Yamuah, L., Kumar, R., Engers, H. and Aseffa, A. (2009) Validation of Bleach-Treated Smears for the Diagnosis of Pulmonary Tuberculosis. The International Journal of Tuberculosis and Lung Disease, 13, 136-141.

[14] Mase, S.R., Ramsay, A., Ng, V., et al. (2007) Yield of Serial Sputum Examinations in the Diagnosis of Pulmonary Tuberculosis: A Systematic Review. The International Journal of Tuberculosis and Lung Disease, 11, 485-495.

[15] Padma, S., Kamesh, S. and Daley, P. (2009) Bleach Optimization of Sputum Smear Microscopy for Pulmonary Tuberculosis. Indian Journal of Tuberculosis, 56, 174.

[16] Pandey, A. and Asthana, A.K. (2009) Sedimentation Method, a Good Alternative to Centrifugation for Concentration of AFB in Developing Countries. Indian Journal of Medical Microbiology, 27, 83-84. 
[17] Best, M., Sattar, S.A., Springthorpe, V.S. and Kennedy, M.E. (1990) Efficacies of Selected Disinfectants against Mycobacterium tuberculosis. Journal of Clinical Microbiology, 28, 2234-2239.

[18] Kent, P.T. and Kubica, G.P. (1985) Public Health Mycobacteriology: A Guide for the Level III Laboratory. Centers for Disease Control, Atlanta.

[19] Lawson, L., Yassin, M.A., Ramsay, A., Olajide, I., Thacher, T.D., Davies, P.D., Squire, S.B. and Cuevas, L.E. (2006) Microbiological Validation of Smear Microscopy after Sputum Digestion with Bleach; A Step Closer to a One-Stop Diagnosis of Pulmonary Tuberculosis. Tuberculosis, 86, 34-40. http://dx.doi.org/10.1016/j.tube.2005.06.003

[20] Ongkhammy, S., Anstutz, V., Baremes, H. and Buisson, Y. (2009) The Bleach Method Improves the Detection of Pulmonary Tuberculosis in Laos. The International Journal of Tuberculosis and Lung Disease, 13, 1124-1129.

[21] Aber, V.R., Allen, B.W., Mitchinson, D.A., Ayuma, P., Edwards, E.A. and Keyes, A.B. (1980) Quality Control in Tuberculosis Bacteriology. Laboratory Studies on Isolated Positive Cultures and the Efficiency of Direct Smear Examination. Tubercle, 61, 123-133. http://dx.doi.org/10.1016/0041-3879(80)90001-X

[22] Yassin, M., Cuveas, A.E.L., Gebrexabher, H. and Squire, S.B. (2003) Efficacy and Safety of Short-Term Digestion of Sputum in Case Finding for Pulmonary Tuberculosis in Ethiopia. The International Journal of Tuberculosis and Lung Disease, 7, 678-683.

[23] Iqbal, S., Amin, D., Mumtaz, A. and Shabir, I. (2010) Significance of Appropriate Sampling in the Diagnosis of Tuberculosis (TB)—A Comparison of Different Techniques. Biomedica, 26, 39-44.

[24] Negi, S.S., Khan, S.F., Gupta, S., Pasha, S.T., Khare, S. and Lal, S. (2005) Comparison of the Conventional Diagnostic Modalities, Bactec Culture and Polymerase Chain Reaction Test for Diagnosis of Tuberculosis. Indian Journal of Medical Microbiology, 23, 29-33. http://dx.doi.org/10.4103/0255-0857.13869

[25] Mindolli, P.B., SalMani, M.P. and ParandeKar, P.K. (2013) Improved Diagnosis of Pulmonary Tuberculosis Using Bleach Microscopy Method. Journal of Clinical and Diagnostic Research, 7, 1336-1338.

[26] James, A., Uba Abba, S., Ibrahim, A., Mbah, H., Musuluma, H., Ochei, K., Darboe, S. and Torpey, K. (2013) Improving the Case Detection of Pulmonary Tuberculosis by Bleach Microscopy Method in the North West of Nigeria. Journal of Medical Laboratory and Diagnosis, 4, 34-37. http://dx.doi.org/10.5897/JMLD2013-0066

[27] Kalawat, U., Sharma, K.K., Reddy, P.N. and Kumar, A.G. (2010) Study of Bronchoalveolar Lavage in Clinically and Radiologically Suspected Cases of Pulmonary Tuberculosis. Lung India, 27, 122-124. 
Scientific Research Publishing (SCIRP) is one of the largest Open Access journal publishers. It is currently publishing more than 200 open access, online, peer-reviewed journals covering a wide range of academic disciplines. SCIRP serves the worldwide academic communities and contributes to the progress and application of science with its publication.

Other selected journals from SCIRP are listed as below. Submit your manuscript to us via either submit@scirp.org or Online Submission Portal.
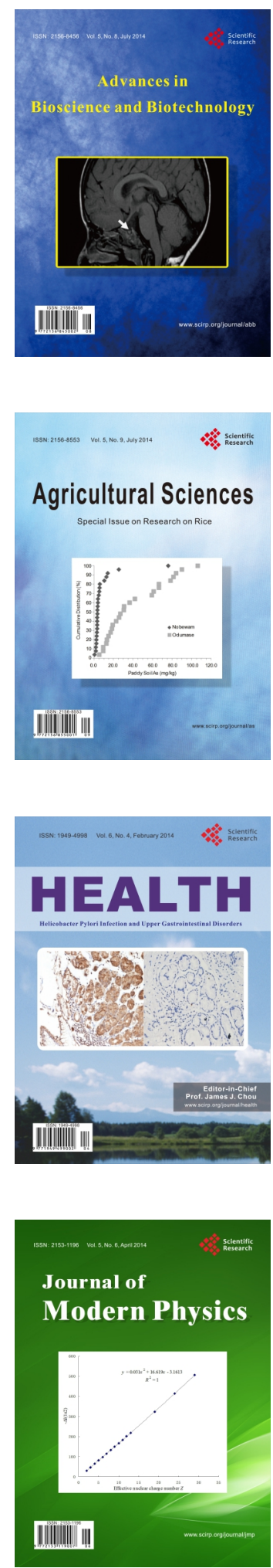
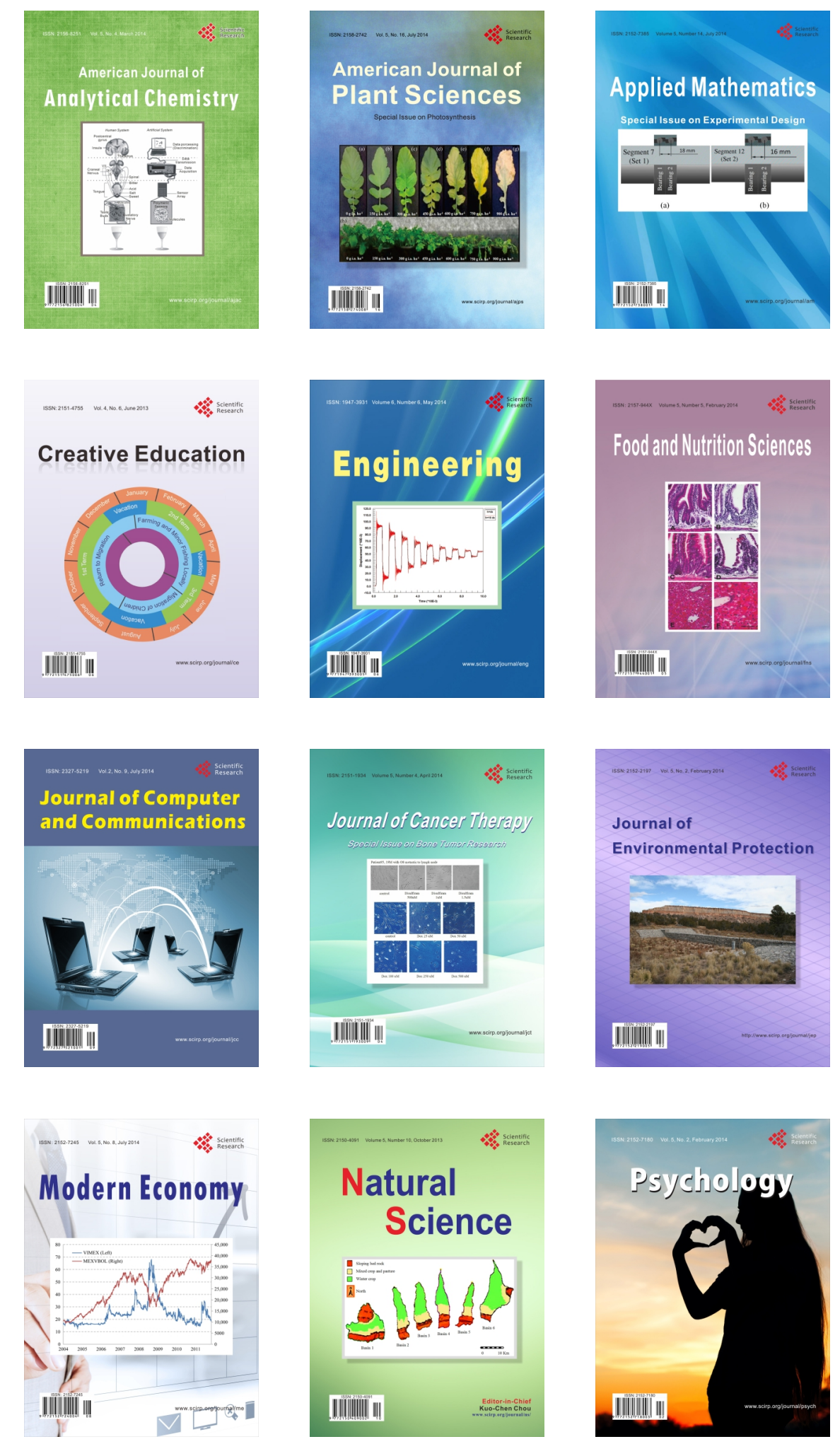